\title{
Anemia status, hemoglobin concentration and outcome after acute stroke: a cohort study
}

\author{
David Tanne*, Noa Molshatzki, Oleg Merzeliak, Rakefet Tsabari, Maya Toashi, Yvonne Schwammenthal
}

\begin{abstract}
Background: In the setting of an acute stroke, anemia has the potential to worsen brain ischemia, however, the relationship between the entire range of hemoglobin to long-term outcome is not well understood.

Methods: We examined the association between World Health Organization-defined admission anemia status (hemoglobin $<13$ in males, $<12 \mathrm{~g} / \mathrm{dl}$ in women) and hemoglobin concentration and 1-year outcome among 859 consecutive patients with acute stroke (ischemic or intracerebral hemorrhage).

Results: The mean baseline hemoglobin concentration was $13.8 \pm 1.7 \mathrm{~g} / \mathrm{dl}$ (range 8.1 - 18.7). WHO-defined anemia was present in 19\% of patients among both women and men. After adjustment for differences in baseline characteristics, patients with admission anemia had an adjusted OR for all-cause death at 1-month of 1.90 (95\% Cl, 1.05 to 3.43$)$ and at 1-year of $1.72(95 \% \mathrm{Cl}, 1.00$ to 2.93$)$ and for the combined end-point of disability, nursing facility care or death of 2.09 (95\% Cl, 1.13 to 3.84) and $1.83(95 \% \mathrm{Cl}, 1.02$ to 3.27) respectively. The relationship between hemoglobin quartiles and all-cause death revealed a non-linear association with increased risk at extremes of both low and high concentrations. In logistic regression models developed to estimate the linear and quadratic relation between hemoglobin and outcomes of interest, each unit increment in hemoglobin squared was associated with increased adjusted odds of all-cause death [at 1-month 1.06 (1.01 to 1.12; $p=0.03$ ); at 1-year 1.09 (1.04 to 1.15; $p<0.01)$ ], confirming that extremes of both low and high levels of hemoglobin were associated with increased mortality.
\end{abstract}

Conclusions: WHO-defined anemia was common in both men and women among patients with acute stroke and predicted poor outcome. Moreover, the association between admission hemoglobin and mortality was not linear; risk for death increased at both extremes of hemoglobin.

\section{Background}

Low hemoglobin, or anemia is a common condition among older adults, with prevalence increasing with age. Anemia is associated with increased mortality [1-4], disability, and poorer physical performance [5] regardless of the underlying cause of the low hemoglobin. In patients with acute coronary syndromes as well as in patients with angina, anemia is an independent indicator of short and long-term mortality [6-8]. At the other end of the range of hemoglobin, patients with an acute coronary syndrome or congestive heart failure with high hemoglobin values also exhibit excess mortality, suggesting a non-linear reverse J-shaped relationship [8,9].

\footnotetext{
* Correspondence: tanne@post.tau.ac.il

Stroke Center, Department of Neurology, the Chaim Sheba medical center, Tel- Hashomer, and the Sackler Faculty of Medicine, Tel Aviv University, Tel Aviv. Israel
}

Among community dwelling elderly, findings are inconsistent but some have observed a similar relationship with mortality $[1,3]$ as well as with a lower level of cognitive function [10].

The relationship between the entire range of hemoglobin and outcome after stroke is not well understood. Given the relevance of hemoglobin to oxygen carrying capacity, inflammatory processes, oxidative stress, as well as to blood viscocity and cerebral blood flow, we hypothesized that extremes of both low and high hemoglobin are associated also with poor outcome in the setting of an acute stroke. Our aims were, therefore, to evaluate in a large cohort of patients hospitalized for an acute stroke: a. the predictive value of admission anemia status; b. the association between the entire range of hemoglobin concentration and stroke outcome. 


\section{Methods}

A prospective cohort study was conducted on 883 consecutive patients hospitalized due to acute stroke in a large medical center with a catchment area of about 500,000 people from March 2001 to June 2002. Baseline hemoglobin was missing in 24 patients, thus the final study cohort included 859 patients. Patients were evaluated systematically for risk factors, stroke severity, type and subtype. Risk factors were assessed from medical records and self-report. Severity of stroke was assessed using the National Institutes of Health Stroke Scale (NIHSS) [11]. Intracerebral hemorrhage and ischemic stroke were differentiated by the results of the baseline head CT scan. Ischemic stroke etiology was determined by the TOAST classification, a system for categorization of subtypes of ischemic stroke mainly based on etiology that has been developed for the Trial of Org 10172 in Acute Stroke Treatment [12]. A clinical evaluation and personal interview was performed after 1-months and a phone follow-up interview after 1-year, after obtaining informed consent. The study was approved by the local Institutional Review Board.

\section{Laboratory methods}

Hemoglobin was measured with the Beckman Coulter MAXM/HMX analyzer (Beckman Coulter, Miami, FL) using the manufacturer's reagents and methods. The first hemoglobin measurement performed at hospital admission served as the index hemoglobin. Anemia was defined by the World Health Organization (WHO) criteria as a hemoglobin concentration $<12 \mathrm{~g} / \mathrm{dl}$ in women and $<13 \mathrm{~g} / \mathrm{dl}$ in men. Chronic kidney disease was defined according to the National Kidney Foundation and American Heart Association with a glomerular filtration rate $<60 \mathrm{~mL} / \mathrm{min} / 1.73 \mathrm{~m}^{2}$, estimated by the 4 variables modification of diet in renal disease equation [13], from baseline serum creatinine measured at a central laboratory using the Jaffe assay.

\section{Outcomes of interest}

Outcomes were assessed during the first year after the stroke. During hospitalization and at 1-month follow-up, data were collected by clinical evaluation and personal interviews with patients and/or proxies. One-year after the stroke, phone follow-up interviews were conducted by professional interviewers from the Israeli Center for Disease Control blinded to in-hospital data. Functional outcome were assessed using the Barthel Index [14] with a score $<75$ regarded as dependent in daily activities. Mortality data were derived from the Israeli Population Registry. The main outcome of interest was all-cause death. In addition, the combined end-point of disability, nursing facility care or all-cause death was assessed.

\section{Statistical analyses}

Anemia was analyzed according to the WHO criteria and hemoglobin concentrations by gender-specific quartiles: First, $<12.3$ in women, $<13.3$ in men; second, 12.313.1 in women, $13.3-14.2$ in men; third, 13.2-14.1 in women, 14.3-15.2 in men; forth, $\geq 14.2$ in women, $\geq 15.3 \mathrm{~g} / \mathrm{dl}$ in men. Unadjusted event rates for endpoints of interest by anemia status and gender-specific quartiles were compared using $\chi^{2}$ test. To examine the relationship between anemia status and outcomes of interest, logistic regression models were conducted. The shape of the relationship between hemoglobin and event rates for end-points of interest was examined by plotting gender-specific hemoglobin quartiles against the percentage of each end-point. The plots showed a quadratic relationship. Then, logistic regression models were developed to estimate the linear and quadratic relation between hemoglobin and outcomes of interest. The variable hemoglobin was centered on its respective mean. All models were adjusted for age, gender, stroke type, stroke severity (NIHSS), prior disability, chronic kidney disease, other cardiac disease and malignancy. To determine whether the relationship of hemoglobin to mortality was influenced by gender, we repeated the adjusted regression models by adding the interaction of each hemoglobin term with gender. To visualize the relation between hemoglobin and all-cause death as demonstrated in the final logistic regression model the Loess method was used. The Loess method is a locally weighted scatterplot smoothing that helps determining the shape of the function that best summarizes the scatter plot between 2 continuous variables [15]. The method requires the input of a "smoothing parameter," which is the fraction of the data that are used around each point. An algorithm for choosing an optimal value for the smoothing parameter according to objective criteria, described by Hurvich and Simonoff [16], was used. For 1 year survival Cox proportional hazard model was used to calculate adjusted HR. All analyses were performed with SAS statistical software version 8.2 (SAS, Inc, Cary, NC).

\section{Results}

The characteristics of the 859 study patients are shown in Table 1 . The mean baseline hemoglobin concentration was $13.8 \pm 1.7 \mathrm{~g} / \mathrm{dl}$ (range 8.1 - 18.7). Age was negatively correlated with hemoglobin $(\mathrm{r}=-0.18, \mathrm{p}<$ $0.01)$, and hemoglobin concentration was lower among women $(13.2 \pm 1.6)$ than among men $(14.2 \pm 1.6 \mathrm{~g} / \mathrm{dl}$; $\mathrm{p}<0.01)$. Hemoglobin was not associated with stroke type or stroke severity, but was lower among those with prior disability, chronic kidney disease, cardiac disease (congestive heart failure or atrial fibrillation or valvular heard disease) and malignancy than those without. 
Table 1 Baseline Characteristics of Study Cohort

\begin{tabular}{lc}
\hline & $\mathbf{N}=\mathbf{8 5 9}$ \\
\hline Age (mean \pm SD) years & $70.6 \pm 12.5$ \\
$\quad$ range & $29-100$ \\
Women & $362(42.2 \%)$ \\
Type of event & \\
$\quad$ Ischemic stroke & $726(84.6 \%)$ \\
$\quad$ Intracerebral hemorrhage & $132(15.4 \%)$ \\
Stroke severity (NIH stroke scale) & \\
$\quad<5$ & $426(50.4 \%)$ \\
$\quad 6$ - 10 & $173(20.5 \%)$ \\
Prior stroke & $247(29.2 \%)$ \\
Prior disability & $232(27.0 \%)$ \\
Hypertension & $265(31.7 \%)$ \\
Dyslipidemia & $605(70.5 \%)$ \\
Diabetes mellitus & $299(34.9 \%)$ \\
Chronic kidney disease & $281(32.8 \%)$ \\
Coronary heart disease (angina pectoris and/or & $305(38.0 \%)$ \\
myocardial infarction) & $268(31.2 \%)$ \\
Other cardiac disease (congestive heart failure, atrial & $226(26.3 \%)$ \\
fibrillation or valvular heart disease) & \\
Peripheral artery disease & $71(8.3 \%)$ \\
Malignancy & $78(9.1 \%)$ \\
\hline Continus vaiabs are expressed as mean \pm SD. Categoical varias &
\end{tabular}

Continuous variables are expressed as mean \pm SD. Categorical variables as number (\%)

WHO-defined anemia was present in $19 \%$ of patients among both women and men.

Mortality data, the main outcome of interest, were available for all patients. At 1-month after the event 728 patients (85\%) were alive, and $586(80 \%)$ of them had a follow-up evaluation. At 1-year, 670 patients (78\%) were alive, and 501 (75\%) of them had a phone follow-up interview. The age, gender and stroke severity of patients with available follow-up data at 1-months and those with available data at 1-year were comparable to the entire study cohort.

\section{Anemia status and clinical outcome}

Clinical outcomes by anemia status are presented in Table 2. Patients with anemia exhibited poorer outcomes at both 30-day and 1-year. Similar associations were observed in men versus women, in younger versus older patients, in patients with ischemic stroke versus intracerebral hemorrhage, with no evidence for an interaction. Because of the imbalances in some baseline characteristics, multivariable logistic regression was used to evaluate the relationship between anemia status with outcomes of interest after adjusting for potential confounders (Table 3). Patients with admission WHOdefined anemia had an adjusted OR for all-cause death after 1-month of 1.90 (95\% CI, 1.05 to 3.43) and after 1year of 1.72 (95\% CI, 1.00 to 2.93 ) and for the combined
Table 2 Clinical Outcomes by Anemia Status

\begin{tabular}{lccc}
\hline End Point & \multicolumn{2}{c}{ Anemia status } & \\
& $\mathbf{n = 1 6 3}$ & $\mathbf{n}=\mathbf{6 9 5}$ & P-value \\
\hline $\begin{array}{l}\text { At 1-Month } \\
\text { All-cause death (\%) }\end{array}$ & $39(23.9 \%)$ & $92(13.2 \%)$ & $<0.01$ \\
$\begin{array}{l}\text { Disability, nursing facility or } \\
\text { death (\%) }\end{array}$ & $89(65.9 \%)$ & $262(44.7 \%)$ & $<0.01$ \\
$\begin{array}{l}\text { At 1-Year } \\
\text { All-cause death (\%) }\end{array}$ & $54(33.1 \%)$ & $135(19.4 \%)$ & $<0.01$ \\
$\begin{array}{l}\text { Disability, nursing facility } \\
\text { or death (\%) }\end{array}$ & $82(66.1 \%)$ & $225(46.3 \%)$ & $<0.01$ \\
\hline
\end{tabular}

Table 3 Adjusted Odds-Ratios (95\% Confidence Intervals) for Outcomes of Interest by Anemia Status

\begin{tabular}{lcc}
\hline & \multicolumn{2}{c}{ Anemia status } \\
End Point & Yes & No \\
\hline At 1-Month & $1.90(1.05-3.43)$ & 1.0 \\
All-cause death (\%) & $2.09(1.13-3.84)$ & 1.0 \\
Disability, nursing facility or death (\%) & & \\
At 1-Year & $1.72(1.00-2.93)$ & 1.0 \\
All-cause death (\%) & $1.83(1.02-3.27)$ & 1.0 \\
\hline Disability, nursing facility or death (\%) & \\
\hline
\end{tabular}

Models were adjusted for age, gender, stroke type, stroke severity (NIH stroke scale), prior disability, chronic kidney disease, other cardiac disease and malignancy.

end-point of disability, nursing facility care or death of 2.09 (95\% CI, 1.13 to 3.84 ) and 1.83 (95\% CI, 1.02 to 3.27) respectively. Adjusted HR for 1-year survival was 1.48 (95\% CI, 1.04 to 2.10 )

\section{Entire range of hemoglobin concentrations and clinical outcome}

The relationships between gender-specific quartiles of hemoglobin and outcomes of interest at 1-year are depicted in Figure 1. Patients with hemoglobin in the bottom quartile exhibited the highest rates of poor outcome. Those in the middle quartiles exhibited the lowest rates, while for patients with hemoglobin in the top quartile, rates of all-cause death increased again. For the combined end-point of disability, nursing facility care or death, patients in the bottom gender-specific quartile exhibited the highest rates while rates were lower across the other quartiles.

Based on our study hypothesis and since the shape of the relationship between hemoglobin and all-cause death showed a quadratic relationship, logistic regression models were developed to estimate the linear and quadratic relation between hemoglobin and outcomes of interest (Table 4). Each unit increment in hemoglobin squared was associated with increased adjusted odds of 


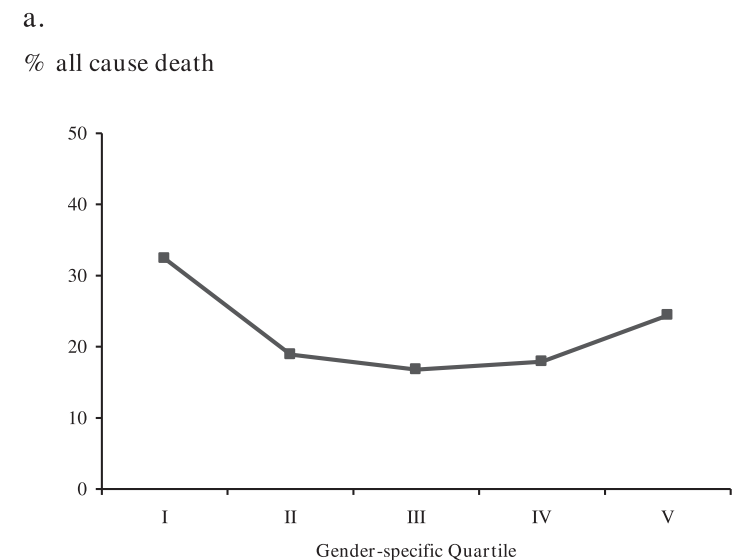

b.

$\%$ disability, nursing facility or death

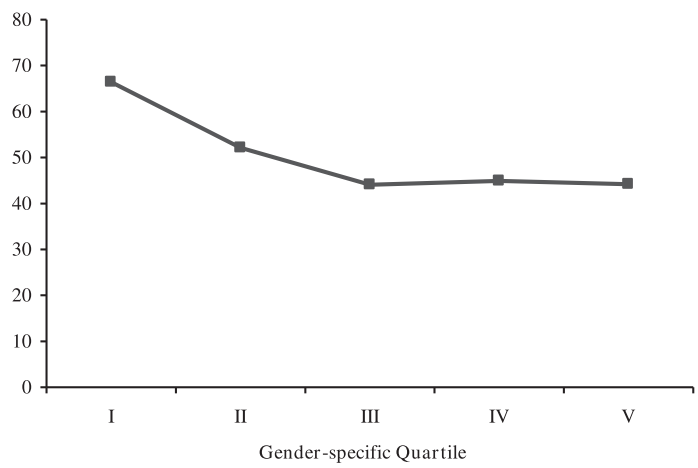

Figure 1 Outcome after 1-year by gender-specific quintiles of admission hemoglobin. a. all-cause death rate; b. disability, nursing facility care or death.

Table 4 Adjusted Odds-Ratios (95\% Confidence Intervals) for Outcomes of Interest by Hemoglobin Squared

\begin{tabular}{lcc}
\hline End Point & Hemoglobin $\times$ Hemoglobin & P-value \\
\hline At 1-Month & $1.06(1.005,1.12)$ & 0.03 \\
All-cause death & $1.07(1.001,1.14)$ & 0.05 \\
Disability, nursing facility & & \\
or death & $1.09(1.04,1.15)$ & $<0.01$ \\
At 1-Year & $1.06(0.998,1.13)$ & 0.06 \\
All-cause death & & \\
Disability, nursing facility & & \\
or death &
\end{tabular}

Models were adjusted for age, gender, stroke type, stroke severity (NIH stroke scale), prior disability, chronic kidney disease, other cardiac disease and malignancy. all-cause death [after 1-month 1.06 (1.01 to $1.12 ; \mathrm{p}=$ 0.03 ); after 1 -year 1.09 (1.04 to $1.15 ; \mathrm{p}<0.01$ )], confirming that extremes of both low and high levels of hemoglobin were associated with increased mortality. Adjusted HR for 1-year survival was 1.05 (95\% CI, 1.02 to $1.09, \mathrm{p}<0.01$ ). As gender may influence the relationship of hemoglobin to mortality, the model was repeated testing for interaction of each hemoglobin term with gender, but no interactions were identified. To visualize the relation between hemoglobin and the probability to all-cause death as demonstrated in the final logistic regression model the Loess method was used. As depicted in Figure 2, the curve reveals a non-linear association with increased rates at both extremes of hemoglobin concentration.

\section{Discussion}

In this cohort of patients with acute stroke followed for 1-year, WHO-defined anemia was common in both men and women, and a significant association was found between anemia and risk for poor outcome. This relationship did not differ between men and women or between patients with intracerebral hemorrhage versus ischemic stroke type. Moreover, the association between admission hemoglobin and mortality was not linear; risk for death increased at both extremes of hemoglobin. Hemoglobin concentrations were inversely correlated with age and were lower among those with prior disability, chronic kidney disease, cardiac disease and malignancy, yet the non-linear association with mortality remained after adjusting for these potential confounders as well as for stroke type and severity.

WHO-defined anemia was present in about a fifth of patients in our cohort, and was a robust predictor of mortality over 1 -year. This finding corroborates a small prior study of 250 ischemic stroke patients, observing that anemia was a negative prognostic factor [17]. Anemia is a particularly common condition among older adults, with prevalence increasing with age. Anemia in the elderly is associated with increased mortality, poorer health-related quality of life, disability, and poorer physical performance [1-5]. Among patients with an acute coronary syndrome, rates of anemia are similar to those observed in our cohort $[8,18]$, and anemia status is associated with poor outcome $[7,8,19,20]$.

High hemoglobin concentrations have been associated with carotid atherosclerosis and may represent a risk factor for ischemic stroke $[21,22]$, but data on their association with outcome after acute stroke are particularly scarce and inconclusive. Sacco et al. found in the population-based L'Aquila registry that high hematocrit might represent an independent predictor of early 
Death Predicted Probability

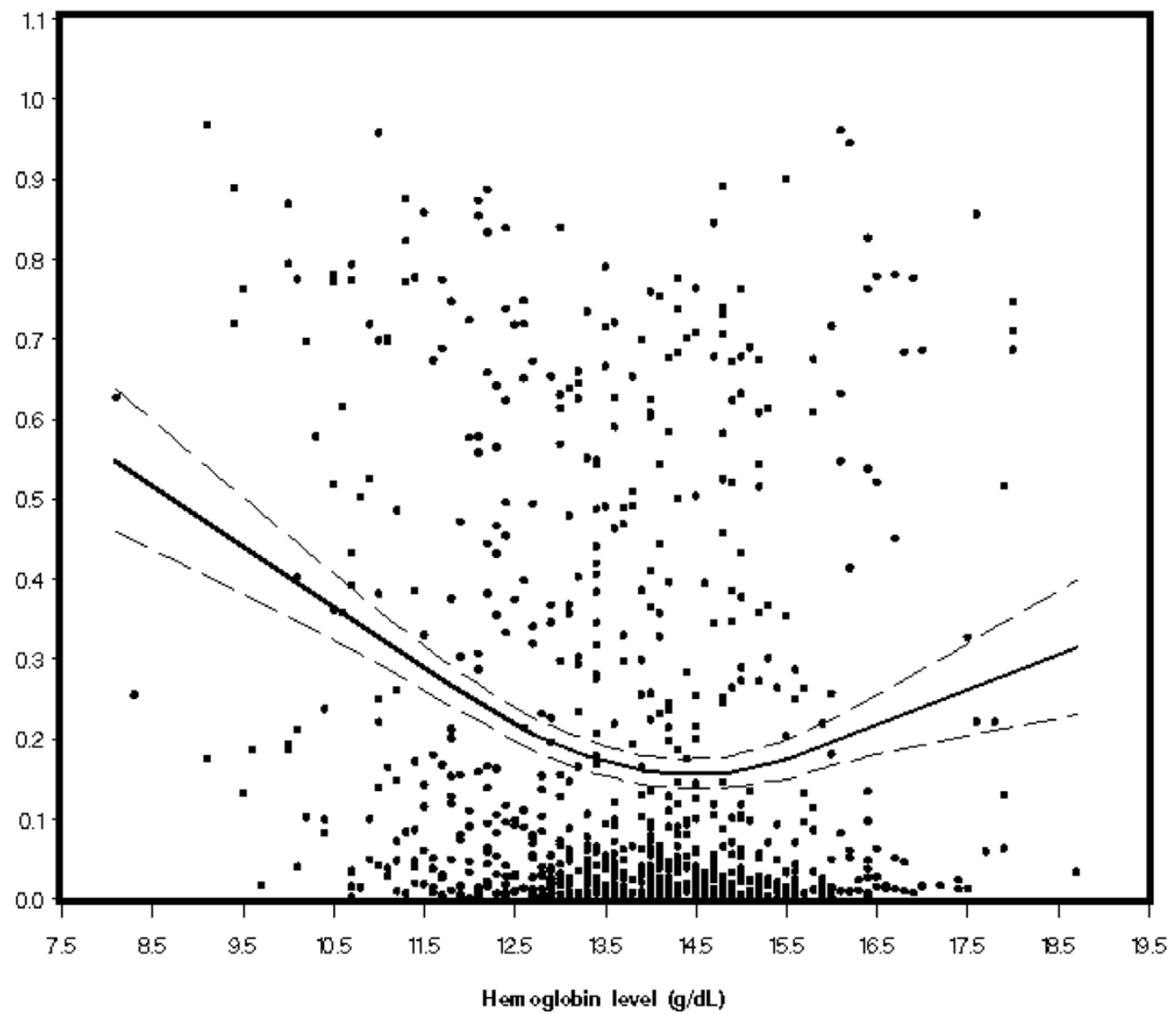

Figure 2 Loess curve (with $95 \%$ confidence intervals) of hemoglobin concentrations versus the estimated probability all-cause death after 1-year

mortality in women with ischemic stroke but not in men [23], In a group of patients with an acute ischemic stroke studied with multimodal magnetic resonance imaging, higher hematocrit had a significant independent association with reduced reperfusion and greater infarct size, suggesting that elevated levels may be a potential physiologic determinant of reduced penumbral salvage [24]. A pilot study on the association between baseline hematocrit at the time of the ischemic stroke and the discharge destination showed that midrange concentrations were associated with a better discharge outcome but were not related to mortality rate [25].

The increased mortality observed in our cohort of stroke patients at both extremes of hemoglobin is in agreement with prior observations in cardiac patients and with some but not all reports among community dwelling elderly $[1,3]$. It remains unclear whether the impact of hemoglobin on mortality after stroke operates through the same or different causal pathways at higher vs. lower values. Anemia may induce hypoxia at the most vulnerable regions when hemoglobin is reduced below a critical level, while high hemoglobin, may increase blood viscosity, affect cerebral blood flow and is associated with pulmonary disease. Moreover, chronically high values might enhance cerebral atherogenesis heading to a diseased and dysfunctional collateral bed [21].

Potential limitations of this study should be considered. First, it is an observational study and therefore causality cannot be inferred from the observed associations. The effects of unmeasured confounding variables such as nutritional status, frailty, cognitive function or complex interactions between covariates on the observed association cannot be ruled out. Second, we were unable to examine whether the relationship was due to increased blood loss, impaired red blood cell production, and/or increased red blood cell destruction (in the case of low hemoglobin levels) or increased red cell production (in the case of high hemoglobin levels). Third, the use of blood transfusions was not 
documented and was based on overall clinical judgment. Admission hemoglobin concentrations were, however, rarely in the range leading to blood transfusion in routine clinical practice. Finally, we did not measure erythropoietin levels in these patients. In addition to stimulating erythrocyte precursors, erythropoietin has multiple neuroprotective effects [26,27], and thus, some of our observations may be due to low or high levels of erythropoietin or other intervening factors rather than hemoglobin per se.

The influence of red blood-cell transfusion on clinical outcomes in selected patients with acute stroke and low hemoglobin has not been tested in trials nor discussed in guidelines. The potential benefits of transfusion by increasing oxygen-carrying capacity must be counterbalanced by risks of acute complications including infection, hemolytic reactions, volume overload, and further activation of adverse biological or inflammatory processes [28]. Achieving higher hemoglobin through pharmacologic treatment with erythropoietin-stimulating proteins was found to increase mortality, at least for anemia due to chronic kidney disease [29]. Hemodilution therapy was not found to improve overall survival or functional outcome in acute ischemic stroke, based on a systematic review of the hemodilution trials [30]. Future areas of investigation should evaluate the causes of increased mortality in individuals with low and high hemoglobin concentrations, and to assess whether aiming to a target range of hemoglobin concentrations may improve long-term outcome after stroke.

\section{Conclusions}

We conclude that WHO-defined anemia is common in both men and women among patients with acute stroke and predicted poor outcome. Moreover, the association between admission hemoglobin and mortality is not linear; risk for death increased at both extremes of hemoglobin. Most importantly, our work suggests that hemoglobin levels (both low and high) may need to be considered as a potential factor in determining outcome after stroke.

\section{List of abbreviations}

NIHSS: National Institutes of Health Stroke Scale; WHO: World Health Organization.

\section{Acknowledgements}

This project was supported by the Israel National Institute for Health Policy and Health Services Research.

\section{Authors' contributions}

DT conceived the study, participated in its design and drafted the manuscript. OM, RT, MT, and YS participated in data collection, interpretation and reviewed the manuscript. NM performed the statistical analysis. All authors read and approved the final manuscript.

\section{Competing interests}

The authors declare that they have no competing interests.

Received: 26 September 2009 Accepted: 9 April 2010

Published: 9 April 2010

\section{References}

1. Zakai NA, Katz R, Hirsch C, Shlipak MG, Chaves PHM, Newman AB, Cushman M: A prospective study of anemia status, hemoglobin concentration, and mortality in an elderly cohort: The Cardiovascular Health Study. Arch Intern Med 2005, 165:2214-2220.

2. Penninx BW, Pahor M, Woodman RC, Guralnik JM: Anemia in old age is associated with increased mortality and hospitalization. J Gerontol A Biol Sci Med Sci 2006, 61:474-9.

3. Culleton BF, Manns BJ, Zhang J, Tonelli M, Klarenbach S, Hemmelgarn BR: Impact of anemia on hospitalization and mortality in older adults. Blood 2006, 107:3841-3846.

4. Dong X, de Leon CM, Artz A, Tang Y, Shah R, Evans D: A population-based study of hemoglobin, race, and mortality in elderly persons. J Gerontol A Biol Sci Med Sci 2008, 63:873-878.

5. Penninx BW, Pahor M, Cesari M, Corsi AM, Woodman RC, Bandinelli S, Guralnik JM, Ferrucci L: Anemia is associated with disability and decreased physical performance and muscle strength in the elderly. J Am Geriatr Soc 2004, 52:719-24.

6. Nikolsky E, Aymong ED, Halkin A, Grines CL, Cox DA, Garcia E, Mehran R, Tcheng JE, Griffin JJ, Guagliumi G, et al: Impact of anemia in patients with acute myocardial infarction undergoing primary percutaneous coronary intervention: analysis from the Controlled Abciximab and Device Investigation to Lower Late Angioplasty Complications (CADILLAC) Trial. J Am Coll Cardiol 2004, 44:547-53.

7. Muzzarelli S, Pfisterer M: Anemia as independent predictor of major events in elderly patients with chronic angina. Am Heart J 2006, 152:991-6.

8. Sabatine MS, Morrow DA, Giugliano RP, Burton PB, Murphy SA, McCabe CH, Gibson CM, Braunwald E: Association of hemoglobin levels with clinical outcomes in acute coronary syndromes. Circulation 2005, 111:2042-9.

9. Go AS, Yang J, Ackerson LM, Lepper K, Robbins S, Massie BM, Shlipak MG: Hemoglobin level, chronic kidney disease, and the risks of death and hospitalization in adults with chronic heart failure: the Anemia in Chronic Heart Failure: Outcomes and Resource Utilization (ANCHOR) Study. Circulation 2006, 113:2713-23.

10. Shah RC, Wilson RS, Tang Y, Dong X, Murray A, Bennett DA: Relation of hemoglobin to level of cognitive function in older persons. Neuroepidemiology 2009, 32:40-6.

11. Brott T, Adams HP Jr, Olinger CP, Marler JR, Barsan WG, Biller J, Spilker J, Holleran R, Eberle R, Hertzberg $V$, et al: Measurements of acute cerebral infarction: a clinical examination scale. Stroke 1989, 20:864-70.

12. Adams HPJ, Bendixen BH, Kappelle LJ, Biller J, Love BB, Gordon DL, Marsh EEd: Classification of subtype of acute ischemic stroke. Definitions for use in a multicenter clinical trial. TOAST. Trial of Org 10172 in Acute Stroke Treatment. Stroke 1993, 24:35-41.

13. Brosius FC III, Hostetter TH, Kelepouris E, Mitsnefes MM, Moe SM, Moore MA, Pennathur S, Smith GL, Wilson PW: Detection of chronic kidney disease in patients with or at increased risk of cardiovascular disease: a science advisory from the American Heart Association Kidney And Cardiovascular Disease Council; the Councils on High Blood Pressure Research, Cardiovascular Disease in the Young, and Epidemiology and Prevention; and the Quality of Care and Outcomes Research Interdisciplinary Working Group: developed in collaboration with the National Kidney Foundation. Circulation 2006, 114:1083-7.

14. Herndon R: Handbook of neurologic rating scales. New York: Demos Vermande 1997.

15. Cleveland WS, Devlin SJ: Locally weighted regression: an approach to regression analysis by local fitting. Journal of the American Statistical Association 83:596-610, CR - Copyright @ 1988, American Statistical Association.

16. Hurvich CM, Simonoff JS, Tsai C-L: Smoothing parameter selection in nonparametric regression using an improved Akaike anformation criterion. Journal of the Royal Statistical Society. Series B (Statistical Methodology) 1998, 60:271-293. 
17. Nybo M, Kristensen SR, Mickley $H$, Jensen JK: The influence of anaemia on stroke prognosis and its relation to $\mathrm{N}$-terminal pro-brain natriuretic peptide. Eur J Neurol 2007, 14:477-82.

18. Alexander KP, Chen AY, Wang TY, Rao SV, Newby LK, LaPointe NM, Ohman EM, Roe MT, Boden WE, Harrington RA, et al: Transfusion practice and outcomes in non-ST-segment elevation acute coronary syndromes. Am Heart J 2008, 155:1047-53.

19. Sarnak MJ, Tighiouart H, Manjunath G, MacLeod B, Griffith J, Salem D, Levey AS: Anemia as a risk factor for cardiovascular disease in The Atherosclerosis Risk in Communities (ARIC) study. J Am Coll Cardiol 2002, 40:27-33.

20. Vaglio J, Safley DM, Rahman M, Kosiborod M, Jones P, Thompson R, Krumholz HM, Spertus JA: Relation of anemia at discharge to survival after acute coronary syndromes. Am J Cardiol 2005, 96:496-9.

21. Irace C, Ciamei M, Crivaro A, Fiaschi E, Madia A, Cortese C, Gnasso A: Hematocrit is associated with carotid atherosclerosis in men but not in women. Coron Artery Dis 2003, 14:279-84.

22. Wannamethee G, Perry IJ, Shaper AG: Haematocrit, hypertension and risk of stroke. J Intern Med 1994, 235:163-8.

23. Sacco S, Marini C, Olivieri L, Pistoia F, Carolei A: Contribution of hematocrit to early mortality after ischemic stroke. Eur Neurol 2007, 58:233-8.

24. Allport LE, Parsons MW, Butcher KS, MacGregor L, Desmond PM, Tress BM, Davis SM: Elevated hematocrit is associated with reduced reperfusion and tissue survival in acute stroke. Neurology 2005, 65:1382-7.

25. Diamond PT, Gale SD, Evans BA: Relationship of initial hematocrit level to discharge destination and resource utilization after ischemic stroke: a pilot study. Arch Phys Med Rehabil 2003, 84:964-7.

26. Grasso G, Sfacteria A, Meli F, Passalacqua M, Fodale V, Buemi M, Giambartino F, lacopino DG, Tomasello F: The role of erythropoietin in neuroprotection: therapeutic perspectives. Drug News Perspect 2007, 20:315-20.

27. Wang L, Zhang Z, Wang Y, Zhang R, Chopp M: Treatment of stroke with erythropoietin enhances neurogenesis and angiogenesis and improves neurological function in rats. Stroke 2004, 35:1732-7.

28. Perrotta PL, Snyder EL: Non-infectious complications of transfusion therapy. Blood Rev 2001, 15:69-83.

29. Singh AK, Szczech L, Tang KL, Barnhart H, Sapp S, Wolfson M, Reddan D, the CHOIR Investigators: Correction of anemia with epoetin alfa in chronic kidney disease. N Engl J Med 2006, 355:2085-2098.

30. Asplund K: Haemodilution for acute ischaemic stroke. Cochrane Database Syst Rev 2002, CD000103.

\section{Pre-publication history}

The pre-publication history for this paper can be accessed here: http://www. biomedcentral.com/1471-2377/10/22/prepub

doi:10.1186/1471-2377-10-22

Cite this article as: Tanne et al:: Anemia status, hemoglobin

concentration and outcome after acute stroke: a cohort study. BMC

Neurology 2010 10:22.

\section{Submit your next manuscript to BioMed Central and take full advantage of:}

- Convenient online submission

- Thorough peer review

- No space constraints or color figure charges

- Immediate publication on acceptance

- Inclusion in PubMed, CAS, Scopus and Google Scholar

- Research which is freely available for redistribution 